\title{
QUOTIENTS OF PERMUTATION GROUPS
}

\author{
JOHN COSSEY
}

\begin{abstract}
If $G$ is a finite permutation group of degree $d$ and $N$ is a normal subgroup of $G$, Derek Holt has given conditions which show that in some important special cases the least degree of a faithful permutation representation of the quotient $G / N$ will be no larger than $d$. His conditions do not apply in all cases of interest and he remarks that it would be interesting to know if $G / F(G)$ has a faithful representation of degree no larger than $d$ (where $F(G)$ is the Fitting subgroup of $G$ ). We prove in this note that this is the case.
\end{abstract}

If $G$ is a finite permutation group of degree $d$ and $N$ is a normal subgroup of $G$, what can we say about the least degree of a faithful permutation representation of the quotient $G / N$ ? Neumann showed in [4] that it could be very much larger than the degree of $G$. Recently Holt [1] has given conditions which show that in some important special cases it will be no larger than $d$. His conditions do not apply in all cases of interest and he remarks that it would be interesting to know if $G / F(G)$ has a faithful representation of degree no larger than $d$ (where $F(G)$ is the Fitting subgroup of $G$ ). Note that we can prove easily from Holt's results that the least degree of a faithful permutation representation of $G / F(G)$ cannot become too large and, in particular, if $n$ is the number of distinct primes dividing $|F(G)|$ then the least degree of a faithful permutation representation of $G / F(G)$ is at most $n d$. To see this, if $\mathcal{S}_{\pi}$ denotes the class of finite soluble $\pi$-groups for a set of primes $\pi$, then two applications of Holt $[1$, Theorem 2] give first that $G / O_{p^{\prime}}(G)$ and then $G / O_{p p^{\prime}}(G)=\left(G / O_{p^{\prime}}(G)\right) / O_{p}\left(G / O_{p^{\prime}}(G)\right)$ have faithful permutation representations of degree at most $d$. It then follows that $G / F(G)$ has a faithful permutation of degree at most $n d$, since if $\left\{p_{1}, \ldots, p_{n}\right\}$ is the set of primes dividing $|F(G)|$ and $X_{i}$ is a set of at most $d$ elements with $G / O_{p_{i} p_{i}^{\prime}}(G)$ acting faithfully, then the natural action of $G$ on $\bigcup_{i} X_{i}$ has kernel $\bigcap_{i} O_{p_{i} p_{i}^{\prime}}(G)=F(G)$. The purpose of this note is to show that we can reduce this bound to $d$.

Received 1st December, 1997

Copyright Clearance Centre, Inc. Serial-fee code: 0004-9729/98 \$A2.00+0.00. 
Theorem. Let $G$ be a finite permutation group of degree $d$. Then $G / F(G)$ has a faithful permutation representation of degree at most $d$.

We prove the theorem by induction on the order of $\left|F(G)^{\prime}\right|$. If $\left|F(G)^{\prime}\right|=1$, then $F(G)$ is a maximal Abelian normal subgroup of $G$ and the result is a consequence of Holt [1, Theorem 1].

We now proceed in a similar fashion to the proof of Holt [1, Theorem 1]. We suppose that $\left|F(G)^{\prime}\right| \neq 1$ and take $N$ to be a minimal normal subgroup of $G$ with $N \leqslant F(G)^{\prime}$. Then $N$ is an elementary Abelian p-group for some prime $p$. Now let $X$ be the set permuted by $G$ and $S$ the symmetric group on $X$. Let $\Sigma$ be the set of orbits of $N$ on $X$ and let $Y$ be a subset of $X$ containing one representative of each orbit $W \in \Sigma$. Let $L$ be the subgroup of $S$ consisting of all $g \in S$ such that for each $W \in \Sigma g$ fixes $W$ and for some $h \in N$ we have $g^{W}=h^{W}$. As in [1], we see that $L$ is an elementary Abelian p-group normalised by $G$. Set $E=G L$ and let $D$ be the setwise stabiliser of $Y$ in $E$. As in [1] we get $E=D L$ and $D \cap L=1$.

Let $\phi$ be the natural homomorphism from $G$ onto $D$ and let $F=\phi(F(G))$. Since the kernel of $\phi, L \cap G$, is Abelian it is contained in $F(G)$ and so $D / F \cong G / F(G)$. In general there is no reason to expect that we shall have $F=F(D)$ (and it is easy to construct examples where $F \neq F(D))$. Our strategy now is to choose a group $H$ between $D$ and $E$ for which $H / F(H) \cong G / F(G)$ and $\left|F(H)^{\prime}\right|<\left|F(G)^{\prime}\right|$.

If $F=F(D)$ we set $H=D$. Now suppose that $F<F(D)$. We have that $F(D) / F$ must be a $p^{\prime}$-group, since otherwise the Sylow $p$-subgroup of $F(D)$ would not be contained in $F$ and so its inverse image, $P$ say, would not be contained in $F(G)$. Since $G \cap L$ is a $p$-group, $P$ is a normal $p$-subgroup of $G$ and so contained in $F(G)$, a contradiction. Let $K$ be the inverse image of $F(D)$ in $G$. Since $L$ is an elementary Abelian $p$-group, we can regard it as a module for $F(D)$ over the field of $p$ elements and then by inflation as a module for $K$. Moreover, since $F(D)$ is nilpotent, we can write $L=L_{1} \oplus \cdots \oplus L_{m}$ as a direct sum of indecomposable modules $L_{i}$ such that all the composition factors of each $L_{i}$ are isomorphic (this is an easy consequence of Huppert and Blackburn [3, Theorem VII.16.10]). Now consider $L \cap G$ as a $K$-module and suppose that the $L_{i}$ have been chosen so that $L_{i} \cap G \neq 0$ for $i=1, \ldots, k$ and $L_{i} \cap G=0$ for $i>k$, so that $G \cap L=\left(G \cap L_{1}\right) \oplus \ldots \oplus\left(G \cap L_{k}\right)$; let $T_{i}$ denote the socle of $L_{i} \cap G, i=1, \ldots, k$. We now have that the action of $K$ on each composition factor of $L_{i} \cap G$ must be the same and further that $K$ centralises each $K$-chief factor of $K /(G \cap L)$. Since $F(G)$ is the Fitting subgroup of $K$ and $K$ is soluble, the intersection of the centralisers of the chief factors of $K$ must be $F(G)$ (Huppert [2, Satz III.4.3]). But then, since the chief factors of $K$ between $G \cap L$ and $K$ are central, we get that the intersection of the centralisers of the composition factors of $L \cap G$ (as $K$-module) must be $F(G)$. It then follows that $F(G)$ is the intersection of the centralisers of the 
$T_{i}, i=1, \ldots, k$ and then that $F$ is the intersection of the centralisers in $D$ of the $T_{i}, i=1, \ldots, k$. We now set $U=T_{1} \times \ldots \times T_{k}$ and note that $F$ centralises each $T_{i}$ and hence $U$. It is easy to check that $U$ is a normal subgroup of $E$. Thus $F U$ is a nilpotent normal subgroup of $D U$. We set $H=D U$ and claim that $F(H)=F U$. To see this observe that $F U \leqslant F(H) \leqslant F(D) U$. Suppose that $F U<F(H)$. Let $Q$ be a Hall $p^{\prime}$-subgroup of $F(H)$; we may suppose that $Q$ is chosen so that $Q \leqslant D$. Since $F(D) / F$ is a $p^{\prime}$-group, $Q F U=F(H)$. But then $Q$ centralises each $T_{i}, i=1, \ldots, k$ and so $F(H)$ is contained in the intersection of the centralisers of the $T_{i}, i=1, \ldots, k$, a contradiction. Thus $F(H)=F U$ and so we have $H / F(H) \cong D / F \cong G / F(G)$. Moreover we have $[U, F]=1$ and so $F(H)=F U=F \times U$. Then we have $F(H)^{\prime}=F^{\prime}$ and since $F$ is a quotient of $G / N$ and $N \leqslant F(G)^{\prime}$ we have $\left|F^{\prime}\right|<\left|F(G)^{\prime}\right|$. Since $H$ has a faithful permutation representation of degree $d$, the result now follows.

The interest of results such as the theorem above is partly in their application in computational group theory. For such applications it is necessary to be able to calculate the permutation representations in the proof explicitly. Apart from the ability to compute the Fitting subgroup of a permutation group, the only extra computational device needed in this proof compared to Holt [1, Theorem 1] is the ability to calculate the socle of a permutation group. We proceed as follows: calculate $F(G)$ and if $F(G)$ is Abelian find $D \cong G / F(G)$ as in [1]. If $F(G)$ is nonabelian, calculate the socle $\sigma(G)$ of $G$ and then find $D$ and $L$ as in the proof above. Next calculate $F(D)$ and then the socle $\sigma(L F(D))$ of $L F(D)$. Set $S=\sigma(L F(D)) \cap L$. Now replace $G$ by $S D$ and repeat.

\section{REFERENCES}

[1] D.F. Holt, 'Representing quotients of permutation groups', Quart. J. Maths. Oxford Ser. (2) 48 (1997), 347-350.

[2] B. Huppert, Endliche Gruppen I (Springer-Verlag, Berlin, Heidelberg, New York, 1967).

[3] B. Huppert and N. Blackburn, Finite groups II (Springer-Verlag, Berlin, Heidelberg, New York, 1982).

[4] P.M. Neumann, 'Some algorithms for computing with finite permutation groups', in Proceedings of Groups-St Andrews 1985, (E.F. Robertson and C.M. Campbell, Editors), London Math. Soc. Lecture Note Series 121 (Cambridge University Press, Cambridge, New York, 1987), pp. 59-92.

Mathematics Department

School of Mathematical Sciences

Australian National University

Canberra ACT 0200

Australia

e-mail: John.Cossey@maths.anu.edu.au 